\title{
Residue on Aryepiglottic Folds Following Swallowing
}

National Cancer Institute

\section{Source}

National Cancer Institute. Residue on Aryepiglottic Folds Following Swallowing. NCI

Thesaurus. Code C127340.

A finding of residue on aryepig lottic folds following swallowing. 